\title{
Cultura material e higiene cotidiana en la Córdoba del Ochocientos/
}

\author{
Material Culture and Daily Hygiene \\ in Córdoba in the Nineteenth Century
}

\author{
Cecilia Moreyra \\ ORCID iD: http://orcid.org/0000-0003-4612-7851 \\ CONICET-CIECS, Argentina
}

Desde la historia de vida cotidiana y los estudios de cultura material, en este trabajo describimos y analizamos las prácticas domésticas de limpieza en la ciudad de Córdoba (Argentina), a partir de la presencia (o ausencia) en los inventarios post mortem de objetos materiales y espacios de la vivienda destinados al aseo. Los cambios en la cultura material y en el espacio construido nos permiten reconocer tanto modificaciones como permanencias en las sensibilidades y en las ideas de limpieza/suciedad.

Palabras Clave: Limpieza/Suciedad; Cultura Material; Vida Cotidiana; Córdoba; Siglo XIX.

From the history of everyday life and material culture studies, in this paper we describe and analyze household cleaning practices in the city of Cordoba (Argentina). We look for the presence (or absence) of material objects and rooms for the daily hygiene in post mortem inventories. Changes in material culture and the domestic architecture allow us to recognize both changes as stays in the sensibilities and the ideas of cleanliness and dirtiness. Keywords: Cleanliness/Dirtiness; Material Culture; Everyday Life; Córdoba; Nineteenth Century.

Copyright: (C) 2017 CSIC. Este es un artículo de acceso abierto distribuido bajo los términos de una licencia de uso y distribución Creative Commons Attribution (CC-by) España 3.0. 


\section{Introducción}

Lejos de ser universales e indistintas para todas las sociedades, las nociones de limpieza y suciedad se construyen y transforman. Los cambios se vinculan al progresivo avance de los umbrales de la vergüenza y el desagrado, inherentes al proceso civilizatorio. ${ }^{1}$ A lo largo de este, según desarrolla Norbert Elias, se añadieron sucesivamente unas exigencias a otras, provocando que determinadas acciones, que entre las sociedades guerreras medievales no generaban repugnancia, sí causen vergüenza o desagrado en los grupos sociales con mayores cadenas de interdependencia y elevada diferenciación de las funciones sociales. En esta línea, una historia de lo limpio/sucio debe tener en cuenta los cambios en las percepciones y sensibilidades de las personas para con sus propios fluidos y olores corporales; el desagrado frente al contacto con el cuerpo de otras personas y sus emanaciones; y también debe reparar en el concepto que se tenga del agua y sus efectos.

En este trabajo proponemos describir y analizar las prácticas domésticas de limpieza en la ciudad argentina de Córdoba a partir de la presencia (o ausencia) de objetos materiales y espacios de la vivienda destinados al aseo. Los inventarios post mortem constituyen una fuente sobradamente eficaz para conocer los objetos que se encontraban en los hogares cordobeses, ya fuera mobiliario, menaje de cocina, servicio de mesa o la ropa de uso de los habitantes de la casa. ${ }^{2}$ Estos documentos oficiales, llevados a cabo luego de un deceso, tenían como fin último la repartición del patrimonio del difunto entre los herederos; para ello, los peritos encargados de realizar la tasación observaban, pesaban y medían cada objeto, consignando no solo el precio sino también las dimensiones, colores, materia prima, ornamentos y estado de conservación de los bienes. La minuciosa lectura y análisis de inventarios post mortem efectuados en la ciudad de Córdoba entre finales del siglo XVIII y finales del XIX, nos permitió identificar los diferentes objetos que utilizaran hombres y mujeres para limpiar sus cuerpos. $\mathrm{Al}$ analizar esta documentación en un período amplio fue posible establecer la frecuencia de algunos artefactos puntuales, es decir la cantidad de veces que determinado

1 Elias, 1988.

2 Existen considerables investigaciones sobre aspectos de la cultura material doméstica que toman a los inventarios post mortem como fuente privilegiada para su análisis (García Fernández, 2003, 2004; Pereira Iglesias y Rodríguez Cancho, 1982, entre otros). Una interesante síntesis de las potencialidades y limitaciones de este tipo de documentación fue puesta de relieve por Sobrado Correa, 2003. 
elemento se menciona en las fuentes a lo largo del tiempo. El definir la frecuencia de tinas de baño, baños, bacinicas y escupideras resultó fundamental para identificar cambios y continuidades en el uso de dichos objetos y en las prácticas cotidianas de aseo. A lo largo de nuestro análisis observaremos que en siglo XIX no solo se incrementó notablemente la cantidad de artefactos destinados a lavar el cuerpo, sino que esos objetos tendieron a ser cada vez más específicos. Lo mismo ocurría con el espacio destinado a la higiene corporal. Progresivamente se asignó un lugar definido dentro de la vivienda para llevar a cabo las prácticas cotidianas de higiene. Estos cambios en la cultura material y en el espacio construido nos permitirán reconocer tanto modificaciones como permanencias en las sensibilidades y en las ideas de limpieza/suciedad.

El espacio temporal que analizamos comprende las últimas décadas del siglo XVIII y se extiende hasta fines de la siguiente centuria. El período resulta significativo por varias razones. Se trata de un espacio temporal plagado de transformaciones políticas y sociales que tocan a diversos espacios del mundo Atlántico. En Latinoamérica se disolvía el edificio colonial dando lugar a la emancipación de los territorios; independencias que fueron seguidas por múltiples conflictos sociales, políticos y militares desatados en torno a la organización política de las regiones ahora independientes de la metrópoli española. Asimismo, la apertura comercial postrevolucionaria significó la masiva llegada de manufacturas provenientes de la Europa industrializada, principalmente Inglaterra. Nuevos y numerosos objetos trajeron consigo nuevas prácticas, nuevas ideas. ${ }^{3}$ Todo ello conformó el tránsito, a distintos ritmos, de una sociedad colonial a una moderna, donde convergieron, convivieron y se superpusieron elementos tradicionales y modernos, nativos y foráneos.

La ciudad de Córdoba ocupaba, desde su fundación en el último tercio del siglo XVI, un lugar periférico dentro de los vastos territorios americanos pertenecientes a la Corona española. En el marco de una economía colonial orientada a la extracción de metálico, las regiones no mineras, como era el caso de Córdoba, orientaban sus actividades productivas y comerciales hacia las regiones donde se extraía metal precioso. El comercio de mulas fue la principal actividad económica de Córdoba durante la colonia

3 Víctor Goldgel (2013) señala la creciente presencia y legitimidad de «lo nuevo» en la primera mitad del siglo XIX hispanoamericano; en medio de transformaciones políticas, tecnológicas, económicas y sociales, y eclipsando otros horizontes de sentido como la tradición y el dogma religioso, lo nuevo se convierte durante esa época en criterio central de asignación de valor. 
y se dirigió, precisamente, hacia el norte minero. Luego de los episodios revolucionarios, Córdoba reorientó su actividad mercantil hacia el espacio Atlántico a través del puerto de Buenos Aires. Observamos, entonces, que aunque periférica, la ubicación de la ciudad de Córdoba era ciertamente estratégica en tanto se constituyó en nudo de rutas comerciales hacia el Alto Perú, Buenos Aires y Cuyo. Por lo tanto, es fácil imaginar una ciudad que a lo largo del período abordado se viera cotidianamente transitada por personas, carretas, mercancías e ideas.

Desde finales del siglo XVIII, cuando el marqués de Sobremonte gobernaba la Córdoba del Tucumán, se llevaron a cabo algunas obras, enmarcadas en el afán modernizador e ilustrado de las reformas borbónicas, que tendieron a mejorar la provisión de agua, controlar las crecidas del río de la Ciudad (actualmente, río Suquía), optimizar el alumbrado, mantener los edificios, establecer un lugar para el faenamiento de animales, y conservar la limpieza de las calles. ${ }^{4}$ Estas acciones no deben concebirse como excepcionales ya que encontramos actuaciones semejantes en otras partes de Latinoamérica, ${ }^{5}$ que respondían a la Ordenanza de Intendentes donde se establecían las condiciones edilicias y de higiene que se debían respetar en las ciudades. ${ }^{6}$

Más adelante, ordenanzas municipales que tuvieron lugar entre 1839 y 1840 disponían la erradicación de las curtiembres ubicadas a la vera del arroyo La Cañada, la prohibición de los entierros en el centro de la ciudad, la restricción del lavado de ropa en las acequias, la organización del riego, la construcción de un camino de entrada a la ciudad y el traslado del cauce del río para evitar las frecuentes inundaciones. ${ }^{7}$ Estas normativas revelan una creciente búsqueda de orden y limpieza en el ámbito urbano. Al establecer un lugar periférico dentro de la traza urbana para determinadas actividades, como la matanza de animales o el curtido de cuero, se pretendía limitar el contacto de la población con desperdicios, olores y demás despojos. Este tipo de reglamentaciones expresan un ideal de orden, una situación deseada, una tendencia en el pensamiento y el discurso, más que constituir

4 Memoria del marqués de Sobremonte escrita para su sucesor el coronel de ingenieros don José González, 1791, en Segreti, 1973, 195-213.

5 Por ejemplo, Ana Inés Punta señala que en Lima, Chuquisaca, Huancavelica y Arequipa, los respectivos gobernadores intendentes instalaron desagües, mejoraron las calles y caminos, se ocuparon de la limpieza e iluminación de los espacios públicos y construyeron hospitales, cárceles y escuelas. Punta, 1997, 177.

6 Ibidem, 178.

7 Ferreira, 1980, 80-94. 
un reflejo de la realidad. Efectivamente, normativas como estas se repiten en el Reglamento de Policía de 1858, donde se establecía la ubicación que debían tener las barracas de cuero, los lavaderos de lana y las curtiembres, consideradas por las autoridades como focos infecciosos y contaminantes. ${ }^{8}$ Los reiterados reclamos por la inobservancia de estas normas que se expresan en la prensa local de finales del siglo XIX, manifiestan lo trabajoso que resultó el proceso de ordenamiento de la ciudad según los modernos preceptos de higiene, salubridad y estética. ${ }^{9}$

Las mencionadas ordenanzas públicas tendientes a ordenar y limpiar la ciudad de Córdoba no fueron inéditas, sino que se enmarcan en un contexto temporo-espacial más amplio. Podemos identificar la base de las preocupaciones higienísticas en la Europa occidental de finales del siglo XVIII. Según analiza Alain Corbin (2002), nuevas prácticas sanitarias, ancladas en conocimientos médicos y científicos, se encaminaron a purificar, es decir, desinfectar y desodorizar, el espacio público parisino. La limpieza de las calles, la desecación de los pantanos pestilentes situados en las cercanías de la ciudad, la limpieza de las letrinas, el destino último de los desperdicios privados. Todo ello, pasó a ser objeto de ordenanzas públicas, de políticas sanitarias que pretendían, más que lavar, ventilar el ambiente, aislar el espacio aéreo de las emanaciones telúricas.

El proceso civilizatorio conllevó cambios en las sensibilidades, en los umbrales de la vergüenza y el desagrado y, a su vez, implicó políticas de salubridad tendientes a limpiar la ciudad, es decir, ordenarla y, con ello, disciplinarla. Es en este amplio marco donde debemos situar algunos de los cambios en las prácticas domésticas de higiene. A ello nos abocaremos a lo largo de este trabajo, el cual se organiza de la siguiente manera: en primer lugar, desarrollaremos los aspectos teóricos y metodológicos de este análisis. La historia de la vida cotidiana y los estudios de cultura material proporcionan los antecedentes y conceptos teóricos referentes de este trabajo. Seguidamente, entrando de lleno en el tema que nos ocupa, nos abocaremos a identificar y analizar las prácticas de aseo en la ciudad de Córdoba, identificando continuidades y transformaciones a lo largo del siglo XIX. Nos concentraremos en cuatro puntos fundamentales, que derivan de los datos provistos por las fuentes: primero, la limpieza en seco y su relación con la muda de ropa, particularmente, la denominada interior o

8 Reglamento de Policía, Córdoba, 1858, Archivo Histórico de la Provincia de Córdoba (AHPC), Sección Gobierno, tomo 5, 22, en Boixadós, 2000, 125.

9 Boixadós, 2000, 124-125. 
«blanca»; en segundo lugar, analizaremos la creciente presencia de tinas, baños y lavatorios que sugieren nuevas concepciones frente al agua y al lavado del cuerpo. En tercer lugar, nos adentraremos en las acciones relativas a la satisfacción de necesidades fisiológicas y, finalmente, describiremos y analizaremos objetos implicados en el arreglo de rostros (navajas de afeitar, escobillas para dientes) y prendas de vestir (cepillos para ropa y bateas de lavar). La progresiva diversificación de instrumentos para limpiar indica una creciente especialización de la cultura material, aspecto característico del período abordado. ${ }^{10}$

\section{Vida cotidiana y cultura material}

En la actualidad existe una amplia producción en torno a la denominada historia de la vida cotidiana, que se enmarca en heterogéneos contextos espacio-temporales y abordan temáticas diversas. Dentro de esta pluralidad, es factible encontrar trabajos descriptivos, algunos analíticos y aquellos que resultan más del tipo «anecdótico», es decir, concentrados en exponer curiosidades y costumbres. Nuestra apuesta es resignificar este ámbito de investigación histórica, atendiendo al gran postulado de la historia cultural: retornar al sujeto. Con todo, aunque nos alejamos del frío determinismo de las estructuras, tampoco pretendemos sobredimensionar la agencia del sujeto, sino que evitamos concebir el proceso histórico desde los extremos, ya fueran las estructuras o las unidades individuales, y buscamos hacerlo, en cambio, desde sistemas de relaciones, entendiendo al individuo y la sociedad en permanente interrelación.

Una de las principales falencias en esa multiplicidad y diversidad de trabajos es el escaso lugar otorgado a la reflexión teórica y la recurrente omisión de una definición de la noción de vida cotidiana. En este sentido, proponemos pensar lo cotidiano como el espacio-tiempo ordinario compuesto de micro experiencias ensambladas, vínculos humanos y vínculos humano-materiales. En su devenir diario, las personas se relacionan entre sí y también interactúan con los diferentes objetos que las rodean. Estas interacciones conforman las vivencias ordinarias (vestirse, comer, dormir,

10 En el caso del mobiliario doméstico, por ejemplo, hemos advertido una contundente diversificación de los muebles de guardado según avanzaba el siglo XIX. Los versátiles baúles donde se guardaban objetos variados y disímiles dieron paso a las cómodas, estantes, roperos y armarios que permitían una mejor organización y acceso a los objetos guardados en estos muebles. Moreyra, 2014a. 
rezar, lavarse, andar, sentarse, entre muchas más); actividades que podemos considerar rutinarias, aunque no por ello espontáneas. De este modo, lejos de ser un ámbito trivial e intrascendente, la vida cotidiana es compleja y está poblada de significados y símbolos.

El análisis de las dimensiones simbólicas y semióticas de los objetos se enmarca dentro de los estudios de cultura material. Este concepto, acuñado desde la arqueología y la antropología, alude a las dimensiones físicas y tangibles de las cosas pero, sin detenerse ahí, ahonda en la permanente interacción que existe entre estas y las personas que las construyen, las piensan, las comparten, las venden, las compran, las utilizan, las desechan. Arqueólogos, geógrafos, arquitectos, artistas, antropólogos e historiadores se han interesado, en las últimas décadas, por el lugar que lo material ocupa dentro de sus respectivos campos de estudio. El hecho de que atraviese diferentes disciplinas da cuenta de la amplitud de este campo de investigación, donde no se pretende abordar los objetos como algo contrapuesto a lo social o lo económico, sino a partir de una relación dialéctica entre sujeto, objeto y sociedad. Desde los clásicos trabajos de Fernand Braudel (1984), Arjun Appadurai (1991), Sindey Mintz (1996) y Daniel Roche (1989), los historiados vienen observando a objetos y mercancías como actores y motores de los procesos históricos. En el espacio latinoamericano, el trabajo de Arnold Bauer (2001) constituyó la primera gran síntesis de las transformaciones en el ámbito de la cultura material. Esta obra constituye todo un referente en este campo de investigaciones, particularmente por proponer un abordaje complejo que tiene en cuenta los aspectos simbólicos de los objetos, problematizando constantemente las razones del consumo.

En tanto nuestro trabajo procura relacionar la cultura material con las prácticas de higiene, resulta imperativo referirnos a Norbert Elias, quien construyó una historia de objetos cotidianos como el tenedor, el pañuelo de nariz y la vestimenta para dormir, entendidos como elementos sustanciales en la conformación de una sociedad «civilizada». El sociólogo alemán explicó los cambios en las sensibilidades y los umbrales de la vergüenza y el desagrado a partir de la aparición, el uso y las reglas establecidas en torno a aquellos objetos. Así, los manuales de urbanidad, por ejemplo, recomendaban usar un pañuelo para recoger las mucosidades de la nariz, evitando que entrara en contacto con otras personas y aconsejaban emplear el tenedor para servirse la comida en lugar de hacerlo con la mano. Siguiendo la línea desarrollada por Elias, Georges Vigarello (1991) analizó, entre las sociedades occidentales, la construcción histórico-cultural de las prácticas 
y percepciones relativas al aseo corporal, el cual pasó de insertarse en el terreno de la civilidad y moralidad a formar parte del ámbito de la salud, y con ello, de la medicina, cobrando entidad el moderno concepto de higiene y con ello, las políticas higienistas y de salubridad que analiza Corbin (2002). La historia de lo limpio y lo sucio habla del perfeccionamiento de la conducta, el aumento del espacio privado y del autodominio, con una creciente relación entre lo íntimo y lo social.

Para identificar cambios en las prácticas domésticas de limpieza en la ciudad de Córdoba, observaremos, tal como anticipamos, la presencia/ ausencia de objetos destinados al aseo de cuerpos, ropas y espacios en los inventarios post mortem. Estos documentos eran llevados a cabo luego de la muerte de una persona, ocasión en que comenzaban a jugar los intereses de distintos actores: la familia, amigos, acreedores y la Iglesia, entre otros. Dependiendo de la complejidad del círculo social del fallecido o fallecida y el tamaño de su patrimonio, las tasaciones, particiones y juicios sucesorios eran más o menos extensos en cantidad de fojas y tiempo insumido en su conclusión. Lo anterior restringe el sector social representado en estos documentos, es decir, que contamos con inventarios cuyos titulares eran, principalmente, hombres y mujeres de la elite socioeconómica. Estos eran personas de etnia española, ${ }_{11}^{11}$ propietarios de tierras, haciendas y solares, dedicados principalmente al comercio y con cargos político-administrativos en la ciudad. Sin embargo, aunque el grueso de la documentación alude a los sectores principales de la sociedad cordobesa, estas fuentes también ofrecen indicios del entorno material de otros sectores, particularmente hombres y mujeres de etnia parda, ${ }^{12}$ algunos libertos, dedicados a la actividad artesanal o al servicio doméstico.

Valiéndonos de una base de datos relacional, ${ }^{13}$ sistematizamos los datos obtenidos en los inventarios. El uso de esta herramienta informática facilitó la consulta de la información y permitió cuantificar algunos objetos, es decir, determinar su frecuencia a lo largo del espacio temporal analizado. No obstante subrayamos la importancia de un análisis cuantitativo, nos

11 Tal como expresa Lockhart $(2000,80)$ el término más adecuado para referirse a las personas que conformaban la cúpula de la sociedad colonial es el de «español/la» en lugar de «blanco/a» ya que este último término raramente aparece en el lenguaje popular u oficial; este grupo conformaba la elite social con poder económico y político, eran los propietarios de esclavos, tierras, haciendas y solares.

12 La denominación «pardo» era ampliamente utilizada para referirse indistintamente a mulatos y mestizos, grupo que tenía el denominador común del tono bronceado de la piel y su dedicación, en la mayoría de los casos, a oficios manuales o mecánicos: alarifes, orfebres, herreros, carpinteros, etc.

13 Construida a partir del programa Microsoft Acces 2007. 
alejamos de propuestas como las de Francisco Aranda Pérez (1991), para quien resulta imprescindible reducir a cifras económicas cada una de las partidas y conceptos con el objetivo de construir una serialización y realizar un estudio estadístico de significación económica. Este tipo de planteo metodológico ignora los detalles y características particulares de cada objeto, que son las que, en definitiva, otorgan significado a lo que de otra manera sería una lista de nombres, números y precios. La realidad no siempre es estadística.

La investigación cuantitativa opera, según Aníbal Bar (2010), en el marco de una lógica conjuntista. Perspectiva que impide captar en su totalidad la complejidad del objeto de estudio, por lo que es imprescindible tener en cuenta que las unidades de análisis son elementos de un complejo mayor, el contexto social cultural, político y económico, que le otorga sentido.

\section{La limpieza en seco}

Observar la vivienda y analizar sus diferentes dependencias constituye una vía conveniente para identificar las experiencias cotidianas que se desarrollaban en los espacios domésticos. La especificidad de algunos cuartos, y el lugar que ocupaban dentro de la traza arquitectónica, dan cuenta de la relevancia que tenían determinadas prácticas en el devenir diario. Las casas que habitaban las familias de la elite cordobesa eran de considerables dimensiones y numerosos cuartos entre los que encontramos sala de recibo, patio principal, alcobas, tiendas, cocina, despensa y cuartos varios. ${ }^{14}$ Cabe mencionar que los espacios estaban fuertemente jerarquizados; en un primer sector, en torno al patio principal se ubicaban las habitaciones más importantes, que eran los lugares donde se movía principalmente la familia dueña de casa. En un segundo segmento de la vivienda, en torno al segundo patio, estaban los espacios de servicio, compuestos por la cocina, pozo de agua, huerta, corral y lugares comunes; en este sector dormían, se movían y vivían los esclavos y personal de servicio.

En la disposición arquitectónica descripta, no se observan espacios específicos para que las personas se higienicen; tampoco había, entre los objetos y muebles que poblaban los diferentes cuartos, elementos tales como tinas o lavatorios. La inexistencia de espacios y artefactos destinados

14 Para esta descripción de las viviendas de la elite cordobesa nos basamos en un trabajo anterior: Moreyra, 2012. 
al baño, insinúa que el cuerpo no se limpiaba a través de la ablución. Esto se enraíza en una mentalidad tradicional reticente al baño que surge y se extiende en la Europa medieval durante la expansión de la peste negra. En ese contexto el agua era concebida como un elemento penetrante, capaz de abrir los poros de la piel dejando a la persona permeable al aire corrupto e indefenso frente a las enfermedades. Así como el agua permitía el ingreso de aire pestilente, también dejaba escapar los humores y vigores. Entonces, el agua enfermaba y debilitaba, desequilibraba y desordenaba. Por todo ello, se desaconsejaba el baño; y al ser los poros considerados la puerta de entrada para los males, solo era posible protegerse mediante ropa de tejidos de tramas compactas. Por lo tanto, para lavar el cuerpo bastaba con limpiar lo que lo envolvía: el vestido. Estas ideas fueron alimentadas por los tratados de urbanidad de los siglos XVII, XVIII y parte del XIX y es factible rastrear su impacto en estas latitudes. Efectivamente, la limpieza personal en la Córdoba de fines del siglo XVIII estaba vinculada a la muda de ropa, tal como observan Vigarello y Sarti ${ }^{15}$ para la Europa moderna. El característico color claro de la ropa interior, hecha en su mayoría con tejidos de lino, explica su denominación en los documentos como «ropa blanca». Blancura que alude a la capacidad que se le atribuía a estas prendas de limpiar el cuerpo del sudor y demás impurezas propias de la piel.

Entre los diversos bienes que constan en las tasaciones, las prendas de vestir ocupan un lugar destacado y, entre estas, las camisas y enaguas femeninas y las camisas y calzoncillos masculinos, están presentes en la mayoría de los inventarios, evidenciando que eran una parte significativa dentro del conjunto de la indumentaria. Incluso se menciona la ropa interior separada del resto de las prendas: «ropa blanca» y «ropa de color» diferenciaban los peritos al inventariar y tasar los bienes del difunto. ${ }^{16}$ Las prendas interiores entraban en contacto directo con el cuerpo absorbiendo, cual esponja, sus secreciones. Por este motivo, para eliminar esa suciedad, era necesario mudarse de ropa; lo que limpiaba no era el agua sino las prendas de vestir. A su vez, aunque las camisas eran prendas íntimas, algunas de sus partes, como el cuello y los puños, podían estar a la vista de los demás. En tanto era importante la apariencia, es decir, lo que el ojo ajeno viera, cuellos y puños

15 Vigarello, 1991, 59-95. Sarti, 2003, 250-254.

16 Los peritos tasadores emplearon el término «ropa blanca» para hacer alusión a la ropa interior tanto de hombres como mujeres, a la que diferenciaron de la «ropa de color» para referirse a las prendas exteriores. Inventario de Manuel Hurquiri, Córdoba, 1811, AHPC, Escribanía 1, leg. 440, exp. 20. Inventario de Antonio Benito Fragueiro, Córdoba, 1813, AHPC, Escribanía 1, leg. 442, exp. 1, 14v. 
eran asunto aun de mayor cuidado que el resto de la prenda. Su candor y sus formas (adornados con encajes y otras guarniciones) indicaban la limpieza general de la persona.

Lejos de ser una actividad realizada a diario, la muda de ropa interior era poco frecuente. En su paso por la campaña rioplatense, el viajero inglés John Miers percibía que «muy pocos lavan o componen sus ropas una vez que se las ponen, las conservan día y noche hasta que se rompen». ${ }^{17} \mathrm{En}$ el reglamento del colegio de niñas nobles huérfanas de finales del siglo XVIII, por ejemplo, estaba dispuesto que las niñas recogidas lavaran y cosieran la ropa que los varones mudaban, o debían mudar, «una vez por semana». ${ }^{18}$ Varios años más tarde, a mediados del siglo XIX, en el Manual de Urbanidad de Manuel Carreño, obra publicada en Caracas pero con una amplia difusión de toda Latinoamérica, se estimaba como conveniente que la muda de ropa se hiciera no menos de dos veces por semana, y si una persona no tenía a su alcance mudar las ropas exteriores que no prescindiera el mudar, al menos, la ropa interior. En ambos casos se trata de prescripciones establecidas, es decir situaciones ideales que se pretendía lograr con estas reglamentaciones, un ideal que probablemente distaba de lo que efectivamente ocurría en la práctica.

\section{La limpieza de los cuerpos se transforma}

A medida que avanzaba el siglo XIX, la higiene en seco se vio si no reemplazada al menos acompañada por el baño, la ablución del cuerpo en el agua contenida en una «tina de bañarse» o en un «baño». ${ }^{19}$ Estos artefactos eran cubos de madera, en el caso de las tinas, o de lata, en el caso de los baños. Medían aproximadamente un metro de largo por unos setenta centímetros de ancho, ${ }^{20}$ lo que nos permite imaginar la postura sentada que se adoptaba dentro de la misma.

17 Miers, 1968, 39, 40, 49.

18 Ghirardi, Celton, Colantonio, 2008.

19 La tercera acepción al vocablo baño que ofrece el primer Diccionario de la Lengua Castellana (conocido como Diccionario de Autoridades) es la siguiente: «Se llama también (por el efecto para que sirve) uno como cubo grande, espacioso y ancho, que de madera o barro y tal vez de metal se hace para bañarse». Diccionario de Autoridades, Madrid, Real Academia Española, 1726, tomo I, 547.

20 La tina para baño que el escribano José Albino Fernández tenía en su vivienda, medía una vara y un cuarto de largo por siete octavas de alto. Inventario de José Albino Fernández, Córdoba, 1827, AHPC, Escribanía 3, leg. 81, exp. 18, 3v. 
Con el correr del tiempo, se incrementó la presencia de tinas y baños en las viviendas, lo que significó que cada vez más personas accedieran a estos dispositivos. ${ }^{21}$ Mientras que en la década de 1810 solo el 7,69 \% de los inventarios refiere una tina de baño, en la década de 1860 aparecen en el 36,36 \% de los documentos. Lógicamente, en el decenio de 1810, cuando la posesión y uso de tinas no era algo extendido, eran las personas de alto estatus socioeconómico quienes empleaban estos elementos especializados, particularmente acaudalados comerciantes. ${ }^{22}$ El baño era un lujo. Las novedades, ya fueran cosas, costumbres o ideas, encontraban lugar primero entre los hombres y mujeres de mayor estatus social, es decir, las personas que tenían el poder adquisitivo para hacerse de las mercancías novedosas y, además, tenían contacto con costumbres e ideas modernas al relacionarse, por ejemplo, con comerciantes europeos. Por el contrario, avanzado el siglo XIX, en la década de 1860 , podemos ver tinas y baños en las viviendas no solo de destacados comerciantes sino también de personas con un perfil profesional y patrimonial más heterogéneo. ${ }^{23}$

La práctica de la ablución del cuerpo entero tenía lugar entre amplios intervalos de tiempo. El lavado de la cara y las manos permitía mantener la limpieza durante esos intervalos o incluso eran un sustituto del baño. Para el lavado diario, las personas se valían de lavatorios, lebrillos o palanganas. Estos artefactos eran vasijas, más o menos profundas, hechas de madera, lata, loza o porcelana, que se ubicaban sobre un cajón, armazón, banco o pie de madera, iban acompañados de la correspondiente jarra que servía para verter el agua dentro de la vasija. En tanto no existía una habitación de la vivienda destinada específicamente para lavarse, este artefacto se situaba en la alcoba o en un cuarto contiguo.

Como en el caso de las tinas y baños, el número de lavatorios registrados en los inventarios creció significativamente a medida que avanzaba

21 El mismo aumento en el número de bañeras es claramente observable en la Francia de finales del siglo XVIII, cuestión que insinúa la paulatina instalación de la práctica del baño relativamente periódico entre la elite francesa de esa época. Vigarello, 1991, 200, 201.

22 Los ejemplares identificados en la década de 1810 estaban en manos de los acaudalados comerciantes Antonio Benito Fraguerio (Inventario de Antonio Benito Fragueiro, Córdoba, 1813, AHPC, Escribanía 1, leg. 442, exp. 1) y José Manrique de Lara (Inventario de José Manrique de Lara, Córdoba, 1818, AHPC, Escribanía 1, leg. 450, exp. 5).

23 Por ejemplo, encontramos una tina para baño entre las pertenencias de Nazario Reta, un pardo libre casado con una esclava liberta (Sucesorio de Nazario Reta, Córdoba, 1863, AHPC, Escribanía 2, leg. 161, exp. 35). Josefa Güemes, una mujer perteneciente al sector artesanal, dedicada a la fabricación de jabón y velas, disponía de una tina y un baño de lata (Inventario de Josefa Güemes, Córdoba, 1863, AHPC, Escribanía 2, leg.129, exp. 8). 
el siglo XIX. En la década de 1810, el 3,85 \% de los documentos menciona alguna forma de lavatorio, mientras que en la década de 1860, fueron identificados en el 52,27 \% de las tasaciones. El incremento de la cantidad, tanto de tinas como de lavatorios, indica que cambiaron las maneras en que se aseaban los cuerpos. Podemos pensar que, progresivamente, las personas dejaban de percibir el agua como un elemento penetrante y corruptor y, en relación a esto, que el aseo personal entró en el terreno de lo íntimo, involucrando a toda la piel que estaba oculta por la ropa. Por mucho tiempo la limpieza del cuerpo y con este, de la ropa, estuvo asociada a ideas de moralidad y civilidad. No es casual, entonces, que quienes se ocuparon de reglamentar estas cuestiones fueran, por largo tiempo, los tratados de urbanidad. Según transcurría el tiempo, y a distintos ritmos, gracias al avance de conocimientos científicos y el espíritu de racionalización que se expandieron en Europa, se comenzó a buscar en el ambiente las casusas de los procesos patológicos, ello llevó a la consideración del aseo de los cuerpos, las casas y la ciudad misma como elementos claves en la prevención de enfermedades. Lo limpio/sucio dejó de ser una mera cuestión de apariencias y comenzó a remitir al ámbito de la salud por lo que las reglamentaciones en torno al aseo pasaron al dominio médico. De este modo, se instalaba el concepto de higiene, entendido como ciencia médica cuyo objetivo era la conservación de la salud. ${ }^{24}$

Cabe aclarar que a pesar de la multiplicación de bañeras y tinas a lo largo del XIX, es probable que las dificultades prácticas de su uso y la mentalidad tradicional que asociaba el baño con la debilidad, se opusieran a estas novedades. Los cambios en las prácticas de higiene no deben ser entendidos en términos revolucionarios, donde una novedosa forma de hacer las cosas reemplazaría definitivamente a las anteriores, sino que diversas prácticas, que remiten a sensibilidades heterogéneas, conviven en un mismo período y un mismo espacio.

A lo largo del siglo XIX se gestó otro cambio relativo al aseo corporal. Recordemos que dentro de la arquitectura doméstica del siglo XVIII y parte del XIX, no había un lugar específico para lavarse. Observemos algunos ejemplos que atestiguan cambios en estas disposiciones espaciales. En la vivienda de Mercedes Bracamonte, una hilandera de etnia española, cuyo inventario se lleva a cabo en 1825 , encontramos que existía, al pie del

24 Recién en 1837 el Diccionario de la Real Academia incluye la definición de la palabra «higiene». Diccionario de la lengua castellana por la Academia Española, Octava edición, Madrid, Imprenta Nacional, 1837. 
brocal del pozo de agua, «un estanco de calicanto para baño». Por otra parte, avanzado el siglo XIX, observamos en la vivienda de los cónyuges Josefa Martínez y Joaquín Urtubey, cuyo inventario data de la década de 1870, la presencia de un pequeño cuarto de baño, que se hallaba en el segundo patio convenientemente ubicado contiguo al pozo de agua. ${ }^{25} \mathrm{El}$ espacio destinado al aseo personal era, en el primer caso, un espacio abierto, un pequeño anexo del pozo de agua, mientras que en la segunda vivienda, el lugar para el lavado del cuerpo era un cuarto separado, con paredes y techo, un sitio definidamente más privado e íntimo. La especialización de los objetos (tinas y baños) y espacios (cuartos de baño) para el lavarse se relaciona con la creciente privatización e individualización de las actividades cotidianas.

Consideramos importante insistir en el carácter procesual de los cambios observados, que a su vez no son en modo alguno casos aislados sino que también pueden advertirse en otros territorios del espacio americano. Para el Medellín de finales del siglo XIX, Luis Gonzáles Escobar se refiere a ciertos cambios en los hábitos privados de limpieza: el aseo del cuerpo y la ropa, la separación del espacio doméstico otorgado a los animales y la clasificación de los espacios interiores. Todo ello enmarcado en prácticas y políticas higienistas importadas de Europa, que se constituyeron en puntos clave del proyecto civilizador de las elites locales. ${ }^{26}$ Recién podemos hablar de higienismo como tendencia coherente de acción social para finales del siglo XIX y comienzos del XX. ${ }^{27}$ Sin embargo, las propuestas de ordenamiento de los espacios urbanos propias de las reformas borbónicas que tuvieron impacto en amplios espacios del territorio americano, son los antecedentes de las acciones salubristas que cristalizarán décadas más tarde.

\section{Las necesidades fisiológicas: bacinicas y lugares comunes}

Las ideas y prácticas relativas a la limpieza/suciedad también se vinculan a todo lo relativo a las evacuaciones diarias de las personas, es decir los desperdicios sólidos y líquidos que los cuerpos generan a diario. Tal como señalamos anteriormente, en las viviendas de la ciudad de Córdoba se evidencia una clara jerarquía de los espacios cotidianos. El sector principal

25 Sucesorio de Josefa Martínez de Urtubey, Córdoba, 1873, AHPC, Escribanía 2, leg. 208, exp. 22, 27-28.

26 González Escobar, 2006.

27 Kingman Garcés, 2006. 
estaba conformado, esencialmente, por el denominado patio principal, el zaguán, la sala de recibo, tiendas y trastiendas y las alcobas de los dueños de casa. En el sector posterior de estas residencias, ya fuera en el segundo patio o traspatio, tenía lugar junto a la cocina, despensa y cuartos para criados, un pequeño espacio llamado «lugares comunes», «secretas» o «necesarias» ${ }^{28}$ constituido esencialmente por un pozo que servía a las funciones de excreción. Los términos empleados para nombrar este particular espacio de la casa, evaden aludir a las «primitivas» actividades que concretamente se desarrollaban a dicho lugar. Más avanzado el siglo XIX, este espacio recibió la denominación de letrina, ${ }^{29}$ término que se utiliza en la actualidad para denominar a los excusados de carácter más precario.

Uno de los elementos clave en el proceso civilizatorio lo constituye el avance del umbral de la vergüenza y el desagrado frente a determinadas prácticas que anteriormente no generaban tal aversión. Las actitudes frente a las necesidades corporales son un claro ejemplo de esta transformación. Los manuales de urbanidad y civilidad minuciosamente analizados por Elias para los siglos XVI, XVII y XVIII establecían como precepto evitar realizar las evacuaciones en escaleras, pasillos o aposentos y hacerlo, en cambio, en los lugares apropiados, que debían estar apartados de la vista de los demás, en lugares «secretos». ${ }^{30} \mathrm{La}$ existencia de este tipo de lugares en las viviendas cordobesas de los siglos XVIII y XIX nos habla de una «domesticación del desperdicio», ${ }^{31}$ donde estos tendrían un sitio determinado dentro del espacio privado; asimismo, sugiere que las funciones fisiológicas estaban excluidas de la vida social, recluidas en un apartado espacio destinado para ello, ubicado en los confines de la casa habitación, lejos de la sala, la alcoba, el comedor y cualquier habitación principal.

Además de un espacio específico, había un utensilio técnico empleado en la satisfacción de las necesidades: la bacinica o bacinilla. Esta podía usarse, por ejemplo, en la alcoba para luego arrojarse su contenido en el correspondiente lugar común, tarea que realizaban, en el caso de haberlos, los esclavos o personal de servicio. Las bacinillas eran instrumentos de forma

28 En el segundo patio de la residencia del comerciante Felipe Antonio González había, junto con despensa, cocina y cuartos para criados, una «necesaria con chimenea de cuatro varas de largo por tres de ancho». Testamentaria de Felipe Antonio González, Córdoba, 1818, AHPC, Escribanía 4, leg. 51, exp.13, 2v.

29 En la espaciosa vivienda de la citada Josefa Martínez de Urtubey había cuatro letrinas. Sucesorio de Josefa Martínez de Urtubey, Córdoba, 1873, AHPC, Escribanía 2, leg. 208, exp. 22, $27-28$.

30 Elias, 1988, 216-229.

31 Laporte, 1989, 33. 
redonda, más o menos profunda, con un asa u «oreja» en el borde que permitía manipularla y solía guardarse en un cajón de madera. ${ }^{32}$ En el Diccionario de 1726 se especifica que este artefacto lo usaban «frecuentemente las mujeres para sus menesteres corporales». ${ }^{33}$ Aunque no sabemos con certeza si en la práctica su uso se limitaba al género femenino, lo cierto es que para los hombres era posible llevar a cabo algunas de sus necesidades estando de pie en casi cualquier lugar, mientras que la posición que debía adoptar la mujer hacía necesario el uso de este instrumento.

La mayoría de las bacinicas registradas en los inventarios eran de plata y loza. ${ }^{34}$ Sin duda, las de plata eran objetos preciados cuyo valor trascendía la estricta función práctica y se las incluía en los inventarios, sobre todo, por el valor que tenía el precioso metal. Las bacinillas de loza, por su parte, comienzan a observarse con mayor frecuencia a principios del siglo XIX, cuando la gran producción de loza inglesa es volcada en los mercados americanos y este material resistente y más barato es empleado para la construcción de vajilla, ornamentos y bacinillas, reemplazando así a otros materiales más caros como la plata, el peltre y las tradicionales mayólicas españolas.

La presencia de bacinillas en los documentos descendió considerablemente con el correr del tiempo. Entre 1810 y 1840, entre el $20 \%$ y el $30 \%$ de los inventarios analizados refiere alguna bacinilla dentro de la lista de bienes. Por el contrario, es nula la presencia de estos utensilios en las tasaciones realizadas entre 1840 y 1870 . El claro descenso del número de bacinillas a lo largo del siglo XIX puede sugerirnos que gradualmente dejaron de usarse y se los reemplazó por otros artefactos con la misma función, o bien tuvieron lugar nuevas prácticas en la satisfacción de las necesidades fisiológicas. Sin embargo, aunque no se las incluía en la tasación de bienes, es probable que las bacinillas continuaran en uso. La exclusión de estos particulares utensilios de la lista de bienes del difunto, ocurría por dos razones fundamentales, por un lado, las bacinillas dejaron de formar parte del preciado conjunto de «plata labrada» y comenzaron a circular y usarse bacinillas hechas con materiales más comunes y baratos como la loza, es

32 Así lo hacía el prestigioso comerciante Hipólito García Posse, al conservar su bacinica de loza catalana dentro de su correspondiente cajón de pino. Sucesorio de Hipólito García Posse, Córdoba, 1821, AHPC, Escribanía 2, leg. 115, exp. 9, 3v.

33 Diccionario de Autoridades, Madrid, Real Academia Española, 1726, tomo I, 528.

34 El $53 \%$ de las bacinillas identificadas en los inventarios de la ciudad de Córdoba entre 1810 y 1870 estaban hechas de plata, el $44 \%$ de loza y un exiguo $3 \%$ de peltre. 
decir que se las excluía por su menor valor económico..$^{35}$ Por otra parte, al menguar la naturalidad con que las personas hablaban de las funciones naturales del cuerpo, decrecía la mención de los objetos relacionados a la satisfacción de las necesidades fisiológicas.

\section{Otros objetos para limpiar}

Inventariadas a la par de otros elementos de aseo, las «navajas de afeitar»o «navajas de barba» eran instrumentos que los hombres usaban para acondicionar su rostro. Se guardaban de a dos, tres o cuatro unidades en sus correspondientes estuches, junto con una navaja de afeitar más angosta llamada verduguillo y acompañadas de las «vasijas de afeitar» y las «piedras de asentar o afilar navajas». Todos estos instrumentos eran adquiridos en los comercios de la ciudad, como la tienda y almacén de Pedro García quien tenía para la venta estuches con navajas de barba a cuatro reales cada uno. ${ }^{36}$ Según Michelle Perrot la diferencia de los géneros masculino y femenino se evidencia en relación a la pilosidad y sus usos: el cabello para las mujeres, la barba para los hombres. Esta puede ser considerada un signo de virilidad, de poder, valor y coraje. ${ }^{37}$ Pero para que el signo de virilidad sea también un signo de civilización esta barba debía domesticarse. De aquí entendemos la importancia que tenían las navajas de afeitar y todos los implementos para domesticar la pilosidad del rostro masculino.

Otro objeto empleado en el aseo personal, que destaca por su singularidad, era la «escobilla para dientes». Si bien constituye un caso excepcional, ya que hemos identificado solo un ejemplar en toda la documentación, vale la pena referirnos a él en tanto encarna la práctica de higiene de una parte específica: los dientes, que también podían limpiarse con los también escasos mondadientes. La escobilla en cuestión pertenecía a Rosa Montes, ${ }^{38}$ una mujer de etnia española y oficio costurera, casada con un comerciante inglés, quien debió haber introducido, entre las diversas mercaderías traídas de Europa, el particular utensilio para la higiene bucal. La importancia de este instrumento se relaciona con la creciente especialización de los

35 Similar es el caso de las prendas de vestir, que a medida que avanzó el siglo XIX dejaron de ser objetos lo suficientemente importantes como para ser tenidos en cuenta a la hora de realizar la lista de bienes del difunto y repartirlos entre los herederos. Moreyra, 2014b.

36 Juicio testamentario de Pedro García, Córdoba, 1855, AHPC, Escribanía 1, leg. 493, exp. 3, 9.

37 Perrot, 2009, 67.

38 Testamentaria de Rosa Montes, Córdoba, 1813, AHPC, Escribanía 3, leg. 61, exp. 4. 
objetos, que sucesivamente son pensados y construidos para cumplir funciones determinadas.

Las denominadas escupideras, otro de los objetos vinculados a la higiene cotidiana, estaban destinadas a contener la saliva que escupían las personas. Eran elementos fundamentales para mantener la limpieza y el orden, en tanto evitaban que se esputara en el suelo de la vivienda. Durante el siglo XVI, según desarrolla Elias, creció en la sociedad occidental el sentimiento de desagrado frente a la emanación de los fluidos corporales. La costumbre de escupir en la calle, la mesa o el piso de la vivienda resultó cada vez más desagradable. En este sentido «la escupidera, como instrumento para combatir ese hábito tiene una gran importancia en el siglo XIX en el interior de las casas, en correspondencia con el avance de los límites de la repugnancia». ${ }^{39}$ En la ciudad de Córdoba, por ejemplo, el comerciante y funcionario público Pedro Funes tenía entre sus bienes una «salivadera de sala»;40 asimismo Bernardo Capdevila, acaudalado comerciante, contaba con una escupidera «de sala». Ambos ejemplos indican que este objeto se ubicaba en la habitación principal de la vivienda, el lugar que ocupaban no solo los dueños de casa sino también las visitas. Esto quiere decir que no permanecía oculta en lugares privados. Escupir, siempre que se usara la salivadera correspondiente, no era considerado un acto desagradable que debía hacerse en «secreto».

A lo largo del siglo XIX es notable la frecuencia de escupideras dentro de los inventarios: en la década de 1810, solo el 3,85\% de los documentos refiere una salivadera o escupidera, mientras que en la década de 1850 el $37,24 \%$ de los inventarios registra un ejemplar de estos. El rotundo incremento en la cantidad absoluta de escupideras manifiesta que se extendió el uso de estos objetos entre los habitantes de la ciudad de Córdoba. A su vez, este aumento de la cantidad de escupideras testimonia la cada vez más extendida práctica de mascar tabaco que luego era arrojado en el recipiente. Con el correr del tiempo, las salivaderas dejaron de usarse; basta observar el entorno material de nuestras sociedades contemporáneas para darnos cuenta de que las escupideras son casi inexistentes. Según Elias, estos artefactos dejaron de usarse porque desapareció en forma más o menos completa la costumbre de escupir o la necesidad de hacerlo, lo que constituye un buen ejemplo de la maleabilidad del espíritu humano. ${ }^{41}$

39 Elias, 1988, 246.

40 Sucesorio de Pedro Funes, Córdoba, 1856, AHPC, Escribanía 2, leg. 152, exp. 28.

41 Elias, 1988, 248. 
¿Qué podemos decir en cuanto a la higiene de las prendas de vestir y los espacios? En los inventarios escasea la mención a elementos destinados a limpiar muebles u objetos, probablemente porque los trapos y demás elementos usados para ello fueran de escaso o nulo valor económico. Esto explicaría su ausencia dentro de las tasaciones. No obstante, se identificaron algunas pocas escobas con las que se barría el suelo enladrillado de las habitaciones más importantes y los pisos de tierra propios de cuartos como la cocina. Estos instrumentos aparecen en pocos inventarios, lo que podría indicar que no era generalizado su uso. Sin embargo, las escobas formaban parte del stock de mercaderías que se vendían en los comercios locales, como en el almacén de Carlos Bardecio, quien tenía como mercadería para la venta 116 «escobas norteamericanas ordinarias». ${ }^{42}$ En este sentido, entendemos que el uso de las escobas era más extendido de lo que reflejan los inventarios, lo que sugiere que estos artefactos eran de corto valor y su inclusión en las tasaciones de bienes era excepcional.

En un período en el que convivieron las prácticas de limpieza en seco con la ablución del cuerpo, el lavado de la ropa era importante. Diferentes elementos servían a estos fines; entre los más comunes estaban las bateas de lavar, los jabones, cenizas o lejía, y las pailas para hacer almidón. La diversidad de objetos para lavar las prendas de vestir sugiere la realización de distintos procesos. Las bateas de lavar y el jabón permitían el lavado general de la ropa. Los bateones o batellones para lejía eran empleados para «hacer la colada», proceso que incluía una mezcla de agua caliente y ceniza «reciente y limpia de carbón» en un cubo o batea que se cubría con un lienzo, luego de pasar la ropa por esta solución recién se la enjabonaba. ${ }^{43} \mathrm{El}$ aumentativo del sustantivo bateón alude al considerable tamaño que debió tener este instrumento, volumen necesario para que cupiera toda la ropa que se deseaba lavar. El proceso general del lavado requería el tiempo necesario para recolectar la ceniza, por un lado, y sacar agua del pozo y ponerla a calentar en la caldera, por otro, sin olvidar que en muchos casos el lavado de la ropa se realizaba directamente llevando las prendas al río.

Las pailas de hacer almidón ${ }^{44} \mathrm{o}$ bien la olla con idéntica función $\mathrm{n}^{45}$ constituyen indicios de otro de los procesos llevados a cabo para acondicionar

42 Sucesorio de Carlos Bardecio, Córdoba, 1865, AHPC, Escribanía 2, leg. 163, exp. 18, 3.

43 Manual de la criada económica, 2010, 191-210. Esta obra fue editada originalmente en Madrid en 1830 y reeditada tres años más tarde en Buenos Aires en la imprenta de la Gaceta Mercantil.

44 Testamentaria de Pedro Rodríguez, Córdoba, 1813, AHPC, Escribanía 3, leg. 61, exp. 1, 15v

45 Inventario de Francisco Castellanos, Córdoba, 1867, AHPC, Escribanía 4, leg. 123, exp. 36, 4. 
los textiles: el almidonado. Procedimiento realizado para dejar la ropa más o menos tiesa y evitar así que se arrugue fácilmente, mejorando su aspecto estético. Luego de realizado el lavado o la «colada» y el almidonado de las prendas, el proceso de dejar lista la ropa incluía, finalmente, el estirado o planchado; para lo que se usaba una plancha para ropa, que debía calentarse con brasas. Todas estas actividades implicadas en el lavado y acondicionamiento de la ropa, requerían muchos brazos para ser llevadas a cabo e insumían una importante cantidad de tiempo. En consecuencia, no cabe duda que los tratamientos realizados para mantener y dejar la ropa en condiciones eran privativos de aquellas personas que podían contar con personal para el trabajo y prendas de repuesto mientras se concluía el lavado, almidonado y planchado de la ropa. No queremos decir en absoluto que las personas de menores recursos no lavaran sus ropas, pero sí es probable que no sometieran sus prendas a semejantes procedimientos que, además de costosos, insumían mucho tiempo. Por este motivo, en el transcurso cotidiano, la ropa era puesta a punto mediante el cepillado, usando los «cepillos de ropa», instrumentos recurrentemente citados en las listas de bienes.

\section{Conclusiones}

A lo largo de este trabajo, nos adentramos en el escenario de la vida privada de los cordobeses del siglo XIX observando un peculiar aspecto de su cotidianidad: el aseo. Este se relacionaba con las percepciones que las personas tenían respecto a su propio cuerpo y los fluidos y olores que este emanaba y, por otro lado, se vinculó fuertemente a las concepciones que se tenían en torno al agua y los efectos que esta podía tener sobre la piel y el cuerpo. El camino que elegimos para estudiar las prácticas de limpieza fue observar, cuantificar y analizar los objetos materiales utilizados para lavar cuerpos, cosas y espacios, a partir del cuidadoso análisis de inventarios post mortem. En este punto cabe recapitular dos cuestiones metodológicas relevantes para este trabajo. Por un lado, reconocemos que los cambios en el ámbito de las prácticas cotidianas no fueron «revolucionarios», sino que coexistieron distintos modos de pensar y hacer, dando lugar a un eclecticismo de formas tradicionales/coloniales con modernas. Por ello, advertir indicios de cambios y nuevas tendencias requiere situarnos en un mediano y largo plazo. Así también lo entienden Pereyra Iglesias y Rodríguez Cancho (1983) en su análisis de la riqueza campesina en Extremadura, donde 
subrayan la necesidad de atender a un período de larga duración para comprender mejor la evolución y desarrollo de las fortunas campesinas.

Por otra parte, reconocemos que los documentos trabajados solo nos proporcionan una lista de objetos, que aunque detallada no deja de ser una nómina que solo se vuelve significativa al poner en contexto los objetos descriptos, es decir, al situarlos en un concierto más amplio que involucre aspectos políticos, sociales y económicos, no solo en la ciudad de Córdoba sino en otros territorios del mundo Atlántico. En este orden de cosas, resultó fundamental considerar las acciones tendientes a ordenar y limpiar las ciudades europeas y, más adelante, americanas, como parte de proyectos ilustrados, civilizadores y modernizadores del espacio público. Ya observamos que algunas ordenanzas encuadradas en las reformas borbónicas del siglo XVIII avanzaron en la búsqueda de aseo de los espacios urbanos, sin embargo, pasarán muchos años hasta que la ciudad de Córdoba contemple auténticos cambios en infraestructura, por ejemplo en la provisión del agua corriente, ${ }^{46}$ que le permitan acercarse a los ideales higienistas que hacía tiempo venían ganando terreno en Europa. Lo mismo se observa en otras ciudades americanas. En Quito, por ejemplo, el impacto de las acciones higienistas recién se percibe hacia finales del siglo XIX y comienzos del $\mathrm{XX}$, aunque sí es factible reconocer antecedentes de acciones salubristas en algunas propuestas y escritos reformistas del siglo XVIII. ${ }^{47}$

Considerar las acciones, propuestas y políticas públicas tendientes a limpiar, ya fuera lavar o airear, las ciudades, es una de las dimensiones de la historia de lo limpio/sucio. El estudio del desplazamiento de las sensibilidades también debe tener en cuenta las prácticas cotidianas. De allí nuestro interés en observar el espacio doméstico identificando la presencia/ausencia de objetos materiales y ámbitos destinados a la higiene del cuerpo como una vía eficaz para obtener pistas de los hábitos privados de aseo. Tal como manifestamos en los apartados anteriores, la frecuencia de algunos objetos fue variando a lo largo del siglo XIX. El creciente número de tinas, baños y lavatorios sugiere la introducción de otras formas de lavar el cuerpo que convivieron con la denominada «limpieza en seco». No solo creció el número de objetos destinados al lavado del cuerpo sino que también se diversifi-

46 En su análisis del proceso de urbanización, modernización y poblamiento de la ciudad de Córdoba, María Cristina Boixadós (2000) señala que hacia 1880, luego de varios años de deficiencia y precariedad en el abastecimiento de agua, se iniciaron las primeras obras de cañerías domiciliarias que mejoraron notablemente el acceso privado al agua y, con ello, las condiciones de salubridad.

47 Kingman Garcés, 2006. 
caron y especializaron, algo que ocurrió también con el espacio. De carecer de un lugar específico dentro de la vivienda, la práctica del lavado del cuerpo llegó a ocupar un cuarto construido pensado para el desarrollo de esta actividad: el cuarto de baño, con su tina o baño correspondiente. Todos estos cambios se insertan en la búsqueda de confort y, por sobre todas las cosas, privacidad, valores destacados de las sociedades burguesas decimonónicas.

No cabe duda de que lo cotidiano se transforma. Lejos de ser inmutables en todo tiempo y lugar, las prácticas ordinarias, en este caso las relativas a la higiene corporal, cambian a lo largo del tiempo. Estas transformaciones tienen que ver, a su vez, con modificaciones en las sensibilidades personales y sociales. No se trata de cambios concluyentes sino largos y complejos procesos en los que intervienen percepciones individuales, discursos y acciones públicas, la producción y comercialización de nuevos productos y la circulación de estos objetos junto con ideas y prácticas.

Recibido el 18 de agosto de 2015 Segunda versión el 18 de mayo de 2016 Aceptado el 14 de junio de 2016

\section{Referencias bibliográficas}

Appadurai, Arjun (ed.), La vida social de las cosas. Perspectiva cultural de las mercancías, México, Grijalbo, 1991.

Aranda Pérez, Francisco, «Prosopografía y particiones de bienes: una propuesta metodológica para el estudio de las oligarquías urbanas castellanas en la Edad Moderna», Cuadernos de Historia Moderna, 12, Madrid, 1991, 259-276.

Bar, Aníbal, «La Metodología Cuantitativa y su Uso en América Latina», Cinta de Moebio, 37, Santiago de Chile, 2010, 1-14.

Bauer, Arnold, Goods, Power, History. Latin America's material culture, New York, Cambridge University Press, 2001.

Braudel, Fernand, Civilización material, economía y capitalismo, siglos XV-XVIII. Tomo I. Las estructuras de lo cotidiano: lo posible y lo imposible, Madrid, Alianza, 1984.

Boixadós, María Cristina, Las tramas de una ciudad, Córdoba entre 1870 y 1895. Elite urbanizadora, infraestructura, poblamiento..., Córdoba, Ferreyra Editor, 2000.

Corbin, Alain, El perfume o el miasma. El olfato y lo imaginario social. Siglos XVIII y XIX, México, Fondo de Cultura Económica, 2002.

Elias, Norbert, El proceso de la civilización. Investigaciones sociogenéticas y psicogenéticas, México, Fondo de Cultura Económica, 1988. 
García Fernández, Máximo, «Extranjeros en la Castilla interior durante el Antiguo Régimen: mentalidad y cultura material: actitudes similares y comportamientos diferenciados», en Villar García, M. B. y Pezzi Cristóbal, P. (eds.), Los extranjeros en la España Moderna, Actas del I Coloquio Internacional, Málaga, Ministerio de Ciencia e Innovación, 2003, tomo II, 241-257.

García Fernández, Máximo, «La cultura material doméstica en la Castilla del Antiguo Régimen», en Sobaler Seco, María de los Ángeles y García Fernández, Máximo (coords.), Estudios homenaje al profesor Teófanes Egido, Junta de Castilla y León, 2004, vol. 2, 249-270.

Ghirardi, Mónica; Celton, Dora y Colantonio, Sonia, «Niñez, Iglesia y "política social". La fundación de Colegio de Huérfanas por el obispo San Alberto en Córdoba, Argentina, a fines del siglo XVIII», Revista de Demografía Histórica, XXVI, 1, Madrid, 2008, 125-171.

Goldgel, Víctor, Cuando lo nuevo conquistó América. Prensa, moda y literatura en el siglo XIX, Buenos Aires, Siglo XXI, 2013.

González Escobar, Luis Fernando, «La "físiología de la ciudad": médicos e ingenieros en el Medellín de hace un siglo», Iatreia, 19, 1, Medellín, 2006, 77-94.

Kingman Garcés, Eduardo, La ciudad y los otros. Quito, 1860-1940. Higienismo, ornato y policía, Quito, FLACSO, 2006.

Laporte, Dominique, Historia de la mierda, Valencia, Pretextos, 1989.

Lockhart, James, «Organización y cambio social en la América española colonial», en Bethel, Leslie (ed.), Historia de América Latina. Tomo 4. América Latina colonial: población, sociedad y cultura, Barcelona, Crítica, 2000, 63-108.

Manual de la criada económica y de las madres de familias que desean enseñar a sus hijas lo necesario para el gobierno de su casa, Córdoba, Buena Vista Editores, 2010. (Edición facsimilar de la primera edición: Madrid, Imprenta de los hijos de doña Catalina Piñuela, 1830).

Miers, John, Viaje al Plata, 1819-1824, Buenos Aires, Solar-Hachette, 1968.

Mintz, Sindey, Dulzura y poder. El lugar del azúcar en la historia moderna, México, Siglo XXI, 1996.

Moreyra, Cecilia, «Imágenes de la vida familiar. Espacios, objetos y rituales cotidianos en Córdoba a fines del siglo XVIII», en Celton, Dora e Irigoyen López, Antonio (eds.), Miradas históricas sobre familias argentinas, Murcia, Editum, 2012, 73-93.

Moreyra, Cecilia, «La casa, los objetos, lo cotidiano. El mobiliario de la sala de recibo en la ciudad de Córdoba, siglo XIX», en Ghirardi, Mónica (comp.), Territorios de lo cotidiano, siglos XVI al XX, Del antiguo Virreinato del Perú a la Argentina contemporánea, Rosario, Prohistoria, 2014a, 247-259.

Moreyra, Cecilia, Cultura material en la ciudad de Córdoba, 1810-1870. Una lectura sociocultural de los objetos cotidianos, Tesis de Doctorado, Universidad Nacional de Córdoba, 2014b. 


\section{CECILIA MOREYRA}

Pereira Iglesias, José Luis y Rodríguez Cancho, Miguel, «Estructura y tipología de las fuentes notariales en Cáceres y su tierra durante los tiempos modernos», Norba. Revista de Arte, Geografía e Historia, 3, Universidad de Extremadura, 1982, 191-203.

Perrot, Michelle, Mi historia de las mujeres, Buenos Aires, Fondo de Cultura Económica, 2009.

Punta, Ana Inés, Córdoba Borbónica. Persistencias coloniales en tiempo de reformas (1750-1800), Córdoba, Universidad Nacional de Córdoba, 1997.

Roche, Daniel, La Culture des apparences. Essai sur l'histoire du vêtement aux XVII e et XVIII ${ }^{e}$ siècles, Paris, Fayard, 1989.

Sarti, Rafaella, Vida en familia. Casa, comida y vestido en la Europa moderna, Barcelona, Crítica, 2003.

Segreti, Carlos, Córdoba. Ciudad y provincia siglo XVI-XX. Según relatos de viajeros y otros testimonios, Córdoba, Junta Provincial de Historia de Córdoba, 1973.

Sobrado Correa, Hortensio, «Los inventarios post-mortem como fuente privilegiada para el estudio de la historia de la cultura material en la Edad Moderna», Hispania, LXIII/3, 215, Madrid, 2003, 825-862.

Vigarello, Georges, Lo limpio y lo sucio. La higiene del cuerpo desde la Edad Media, Madrid, Alianza, 1991. 